\title{
Desarrollo de catalizadores sólidos tipo zeolíticos para valorización de derivados de biomasa
}

\section{Development of solid zeolitic catalysts for biomass valorization reactions}

Presentación: 11/10/2019

Doctorando:

\section{Luciana Bonetto}

Centro de Investigación y Tecnología Química (CITeQ)- UTN- CONICET

lbonetto@frc.utn.edu.ar

\section{Director/es:}

\section{Dra. Clara Saux y Dra. María Soledad Renzini}

\section{Resumen}

El objetivo principal de este plan de tesis es el desarrollo de zeolitas micro/mesoporosas mediante síntesis directa y tratamientos alcalinos post síntesis para su aplicación en procesos catalíticos de valorización de biomasa. En este trabajo se propone la generación de porosidad adicional en zeolitas microporosas con estructura ZSM-5 y ZSM-11 mediante síntesis directa empleando almidón soluble y un surfactante catiónico (CTAB) como plantillas mesoporosas. Se estudió el efecto del tiempo de cristalización y la modificación del contenido de almidón y CTAB en la síntesis sobre la obtención de la estructura cristalina y la generación de mesoporosidad en los materiales. Los sólidos obtenidos fueron caracterizados por diferentes técnicas como difracción de rayos X (DRX), Isotermas de adsorción y desorción de nitrógeno, área superficial BET, Microscopía electrónica de barrido (SEM), Espectroscopía de emisión atómica con plasma inductivamente acoplado (ICP- AES). Los resultados confirmaron que las zeolitas obtenidas por ambas síntesis poseen microporos y mesoporos en su estructura.

Palabras claves: zeolitas, jerarquización, síntesis directa, plantillas mesoporosas, métodos post síntesis.

\begin{abstract}
The main objective of this thesis plan is the development of micro/mesoporous zeolites through direct synthesis and post synthesis alkaline treatments for their use as heterogeneous catalysts in biomass valorization reactions. In this work we propose the generation of additional porosity in microporous zeolites with ZSM-5 and ZSM-11 structures by direct synthesis using soluble starch and a cationic surfactant (CTAB) as mesoporous templates. The effects of the soluble starch and $\mathrm{CTAB}$ contents and crystallization times on the crystalline structure and mesoporous generation were studied. The obtained solids were characterized by X-ray diffraction (XRD), nitrogen adsorption and desorption isotherms, BET surface area, scanning electron microscopy (SEM) and inductively coupled plasma atomic emission spectroscopy (ICP-AES).The results confirmed that the obtained zeolites by both synthesis possess micro and mesoporous in their structure.
\end{abstract}

Keywords: Zeolites, Hierarchical, direct synthesis, mesoporous templates, post synthesis methods. 


\section{Introducción}

Las zeolitas son aluminosilicatos cristalinos microporosos compuestos por óxidos de silicio y óxidos de aluminio tetraédricamente coordinados que forman una red tridimensional con forma y tamaño de poros uniforme. Estos materiales poseen una amplia variedad de propiedades fisicoquímicas, tales como elevada área superficial, fuerte acidez, alta estabilidad térmica e hidrotermal, selectividad de forma, entre otras. Como consecuencia de sus excelentes propiedades y características, las zeolitas vienen siendo empleadas como adsorbentes industriales y catalizadores heterogéneos en industrias petroquímicas y refinerías gracias al aumento de la eficiencia de estos procesos y a la reducción de los efectos no deseados a nivel medioambiental. Sin embargo, presentan problemas difusionales para moléculas voluminosas, ya que su diámetro de poro $(<2 \mathrm{~nm})$ restringe el acceso a los sitios activos de estos catalizadores.

Por este motivo, surge la necesidad de desarrollar materiales con porosidad adicional, en el rango de los mesoporos, conservando las características y propiedades fisicoquímicas inherentes a las zeolitas microporosas. Para ello, existen una amplia variedad de rutas de síntesis y modificaciones alcalinas post síntesis que pueden emplearse para lograr la "jerarquización" de las zeolitas microporosas. La generación de mesporosidad a través de la síntesis directa implica el uso de diferentes plantillas mesoporosas, tales como surfactantes catiónicos (CTAB) [1-3] y plantillas supramolecuales. Las plantillas supramoleculares tienen las ventajas de ser no tóxicas, inofensivas, inocuas y de bajo costo. Dentro de las mismas el almidón soluble, la glucosa o el alginato de sodio pueden ser incorporados en el gel de síntesis durante el tratamiento hidrotermal [4-5]. A su vez, entre las modificaciones post síntesis se pueden mencionar la desilicación, utilizando soluciones alcalinas de distintas bases y sales $\left(\mathrm{NaOH}, \mathrm{KOH}, \mathrm{NH}_{4} \mathrm{OH}, \mathrm{NaCO}_{3}\right)$ [6-7] para generar una destrucción parcial de la red a través del lixiviado del silicio. Finalmente, el método de recristalización implica la desilicación seguida de un tratamiento hidrotérmico en presencia de CTAB.

Por lo expuesto, para el desarrollo de esta tesis de doctorado en ingeniería mención materiales se plantea la preparación de materiales micro/mesoporosos con topología tipo ZSM-5, ZSM-11, Y y/o BETA mediante diversos métodos de generación de porosidad adicional en la estructura zeolítica y su posterior modificación por la incorporación de distintas especies activas $(\mathrm{Cu}, \mathrm{Ru}, \mathrm{Zr}$ y/o Ni). Los materiales sintetizados y modificados serán caracterizados por diversas técnicas y se evaluará su performance catalítica en una serie de reacciones de valorización de derivados de biomasa.

En el presente trabajo se expone la preparación de zeolitas microporosas jerarquizadas con topología, ZSM-5 y ZSM-11 mediante la síntesis directa empleando distintas plantillas mesoporosas y agentes directores de estructura. Por un lado, se sintetizaron zeolitas ZSM-5 micro/mesoporosas mediante tratamiento hidrotermal utilizando hidróxido de tetrapropilamonio (TPAOH) como agente director de estructura y almidón soluble para la formación de mesoporosidad. En esta síntesis se propuso el empleo de almidón soluble por ser un biopolímero natural, abundante económico, no contaminante e inocuo, y se estudió el efecto de su contenido en la creación de mesoporosidad y sobre la estructura cristalina.

Por otra parte, se prepararon zeolitas ZSM-11 con porosidad adicional incorporando bromuro de hexadeciltrimetilamonio o bromuro de cetiltrimetilamonio (CTAB) en el gel de síntesis y utilizando hidroxidotetrabutilamonio (TBAOH) como agente director de estructura. Para esta síntesis, la selección del CTAB se fundamenta en su bajo costo, disponibilidad comercial y alta eficacia en la formación de materiales mesoporosos. En el desarrollo del mismo, se estudió la influencia del tiempo de cristalización en la formación de la estructura cristalina y el efecto del contenido de CTAB en la generación de mesoporosidad.

Los catalizadores micro/mesoporosos obtenidos por los diversos protocolos de síntesis fueron caracterizados mediante diversas técnicas tales como Difracción de rayos X (DRX), Isotermas de adsorción y desorción de nitrógeno, Área superficial BET, Espectroscopía de emisión atómica con plasma inductivamente acoplado (ICP- AES) y Microscopía de barrido electrónico (SEM).

En base a los prometedores resultados alcanzados hasta el momento, se continúa trabajando en la creación de mesoporosidad en las zeolitas ZSM-11 mediante tratamiento alcalino con distintas bases y la recristalización utilizando hidróxido de potasio como solución alcalina y CTAB como plantilla mesoporosa.

\section{Metodología}

Para la obtención de las zeolitas ZSM-5 micro/mesoporosas se procedió a mezclar aluminato de sodio, como fuente de aluminio, hidróxido de tetraetilamonio (TEOS), como fuente de silicio, hidróxido de tetrapropilamonio (TPAOH) con agua destilada a $80^{\circ} \mathrm{C}$ bajo agitación magnética durante $180 \mathrm{~min}$. Posteriormente, para estudiar el efecto del contenido de almidón en la formación de mesoporosidad, se agregaron a la solución anterior distintas cantidades de almidón soluble comercial (2 8 g) previamente disueltos en agua destilada. El gel obtenido se mantuvo con agitación por otros 30 min a la misma temperatura. Finalmente, la mezcla resultante se transfirió a un reactor de acero inoxidable recubierto con camisa de teflón 
bajo presión autogenerada y se mantuvo en estufa a $140^{\circ} \mathrm{C}$ para favorecer el proceso de cristalización por $48 \mathrm{~h}$. Los materiales obtenidos fueron denominados ZSM-5-x, en donde $\mathrm{x}$ indica el contenido de almidón incorporado en el gel de síntesis.

Por otro lado, para la síntesis de las zeolitas ZSM-11 con porosidad jerarquizada se procedió a mezclar aluminato de sodio, dióxido de silicio como fuente de silicio, hidróxido de tetrabutilamonio (TBAOH) con agua destilada bajo agitación a temperatura ambiente hasta homogeneizar la mezcla. La misma se añadió a una solución acuosa de CTAB con $\mathrm{NaOH}$ y se mantuvo con agitación a temperatura ambiente por 2 horas. Posteriormente, la mezcla resultante se transfirió a un reactor de acero inoxidable recubierto con camisa de teflón bajo presión autogenerada y se mantuvo en estufa a $140{ }^{\circ} \mathrm{C}$ a diferentes tiempos para cristalización ( $2-8$ días). Los materiales obtenidos de esta manera fueron denominados ZSM-11-x, en donde x representa el tiempo de cristalización. Para estudiar el efecto del contenido de CTAB en la creación de mesoporosidad, se trabajó con diferentes cantidades de este surfactante incorporado en el gel de síntesis $(0-1,5 \mathrm{~g})$ en un período de cristalización de 4 días. Estos materiales se denominaron ZSM-11-4-y, en donde y indica el contenido de CTAB en el gel de síntesis.

Los productos sólidos obtenidos en estos procedimientos fueron filtrados y lavados con agua destilada hasta alcanzar $\mathrm{pH}$ neutro; desorbidos con flujo de nitrógeno y finalmente calcinados en mufla a $500^{\circ} \mathrm{C}$ por 8 horas para remover los agentes directores de estructura y las plantillas mesoporosas ocluídas en la estructura zeolítica.

\section{Resultados}

Con el objeto de evaluar el efecto del contenido de almidón sobre las características texturales y morfológicas de los materiales zeolíticos del tipo ZSM-5 se presentan en la Figura 1 los patrones de difracción de rayos X (DRX) y en la Figura 2 las isotermas de adsorción y desorción de nitrógeno de los materiales obtenidos.

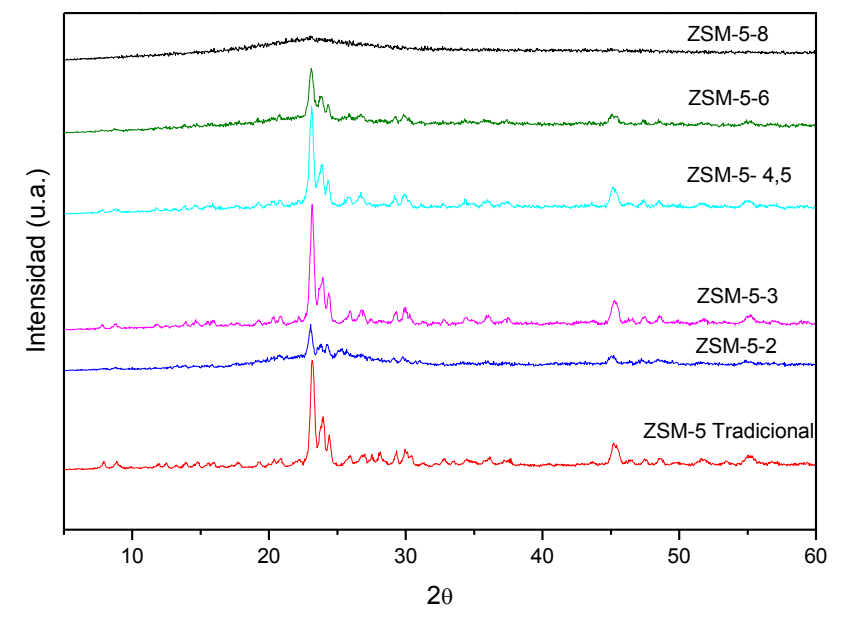

Figura1. DRX de los materiales preparados en función del contenido de almidón.

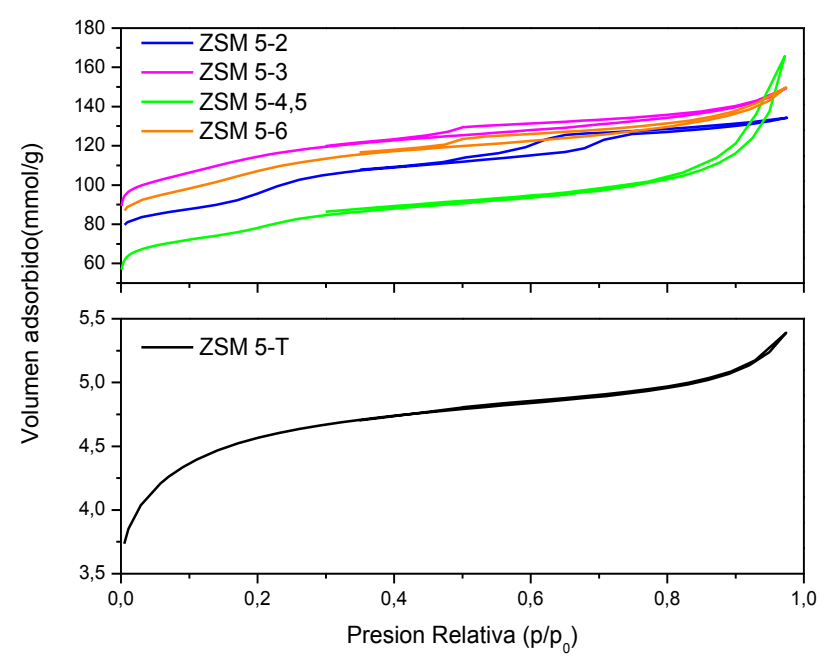

Figura2. Isotermas de adsorción y desorción de $\mathrm{N}_{2}$.

Como se observa en la Figura 1, las zeolitas sintetizadas con un máximo de $6 \mathrm{~g}$ de almidón soluble poseen estructura cristalina, puesto que exhiben los picos de difracción característicos de las zeolitas con topología ZSM-5 bien resueltos. En el caso del material obtenido al incorporar $8 \mathrm{~g}$ de almidón en la síntesis (ZSM-5-8) presenta el patrón característico de un sólido amorfo.

A su vez, en la Figura 2 se observa que las zeolitas ZSM-5 tradicional exhibe isotermas tipo I característica de materiales microporosos. Se graficó su isoterma en un gráfico individual para poder observar sus características, puesto que el volumen adsorbido fue considerablemente inferior al de los materiales jerarquizados. Cuando se incorporó almidón en el gel de síntesis, los materiales presentaron isotermas combinadas tipos I y IV con variación en el lazo de histéresis según el contenido de almidón confirmando la presencia de micro y mesoporos en la estructura zeolítica. En la Tabla 1 se resumen las propiedades texturales de los sólidos obtenidos en función del contenido de almidón soluble. 
Tabla 1: Propiedades texturales de los materiales preparados.

\begin{tabular}{cccccc}
\hline Catalizador & $\begin{array}{c}\mathrm{S}_{\mathrm{BET}} \\
\left(\mathrm{m}^{2} / \mathrm{g}\right)\end{array}$ & $\begin{array}{c}\mathrm{V}_{\mathrm{TOTAL}} \\
\left(\mathrm{cm}^{3} / \mathrm{g}\right)\end{array}$ & $\begin{array}{c}\mathrm{V}_{\text {MICRO }} \\
\left(\mathrm{cm}^{3} / \mathrm{g}\right)\end{array}$ & $\begin{array}{c}\mathrm{V}_{\text {MESO }} \\
\left(\mathrm{cm}^{3} / \mathrm{g}\right)\end{array}$ & $\begin{array}{c}\mathrm{D}_{\text {PORO }} \\
(\mathrm{nm})\end{array}$ \\
\hline ZSM-5 T & 266,21 & 0,121 & 0,121 & - & 2,8 \\
ZSM-5-2 & 275,34 & 0,213 & 0,056 & 0,157 & 3,01 \\
ZSM-5-3 & 263,21 & 0,231 & 0,105 & 0,126 & 3,51 \\
ZSM5-4,5 & 295,26 & 0,232 & 0,085 & 0,147 & 3,14 \\
ZSM-5-6 & 191,78 & 0,257 & 0,056 & 0,201 & 5,35 \\
\hline
\end{tabular}

De los resultados aquí presentados se evidencia un aumento en la mesoporosidad de las zeolitas que acompaña el contenido del carbohidrato en el gel de síntesis. Dicho incremento se produce a expensas de la reducción de microporosidad en su estructura, puesto que el volumen de microporos de los materiales sintetizados disminuye en comparación con el de la zeolita tradicional. A su vez, el volumen total y el diámetro promedio de poros incrementaron con el contenido de almidón en comparación con el de la zeolita ZSM-5 tradicional. Otro aspecto a destacar es el aumento progresivo del área superficial BET de los sólidos hasta 4, $5 \mathrm{~g}$ de almidón.

Los resultados obtenidos por ICP determinaron que las zeolitas jerarquizadas poseen una óptima relación molar Si/Al próxima a 25 en todos los casos. A su vez, en las micrografías de SEM (no presentadas en este trabajo) se apreciaron con claridad los cristales hexagonales característicos de la morfología MFI de las ZSM-5, con agregación de cristales de menor tamaño. De esta manera, los resultados de DRX, isotermas de adsorción y desorción de nitrógeno, SEM y la caracterización textural ponen de manifiesto la presencia simultánea de micro y mesoporos en la zeolita ZSM-5.

En cuanto a la síntesis con incorporación de surfactantes catiónicos, se evaluó el empleo de CTAB en el gel de síntesis. En la Figura 3 se muestran los patrones de difracción de DRX de las zeolitas ZSM-11 jerarquizadas en comparación con la síntesis microporosa tradicional. En la Figura 3 A se evalúa el efecto del tiempo de cristalización para los materiales sintetizados con $\mathrm{x}=0,4,0,76,1$ y $1,5 \mathrm{~g}$ de CTAB, mientras que en la $\mathrm{B}$ se evalúa el efecto de la cantidad de CTAB incorporado en el gel de síntesis en un tiempo de cristalización de 4 días.
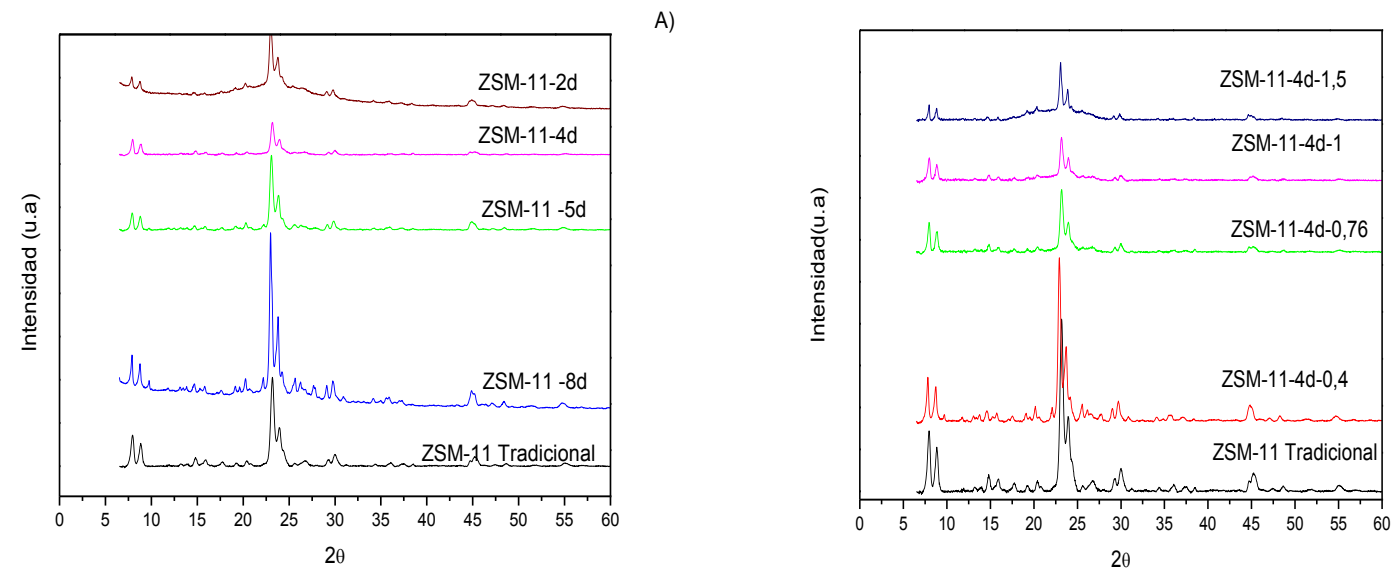

Figura 3. Patrones de difracción de las zeolitas jerarquizadas. A) Efecto del tiempo de cristalización. B) Efecto cantidad de CTAB en el gel de síntesis.

Como se aprecia en la Figura 3 A los materiales obtenidos poseen estructura cristalina, puesto que exhiben los picos de difracción característicos de la zeolita ZSM-11. Sin embargo, los patrones de la zeolitas ZSM-11-4d y ZSM-11-2d mostraron la presencia de una fase amorfa (ensanchamiento de la base de los picos), en comparación con las otras muestras, indicando que la cristalinidad de estos materiales aumenta con el incremento del tiempo de cristalización.

Por su parte, en la Figura 3 B se evidencia que la cantidad de CTAB generó un impacto sobre la cristalinidad de los materiales obtenidos. Tal como se observa en los difractogramas, al aumentar el contenido de CTAB en el gel de síntesis se obtiene una progresiva disminución en las intensidades de los picos, particularmente cuando se los compara con los de la ZSM-11 tradicional.

En la Figura 4 A y B se presentan las isotermas de adsorción y desorción de nitrógeno de las zeolitas jerarquizadas y la ZSM-11 tradicional, al evaluar el tiempo de cristalización y el contenido de CTAB, respectivamente. 

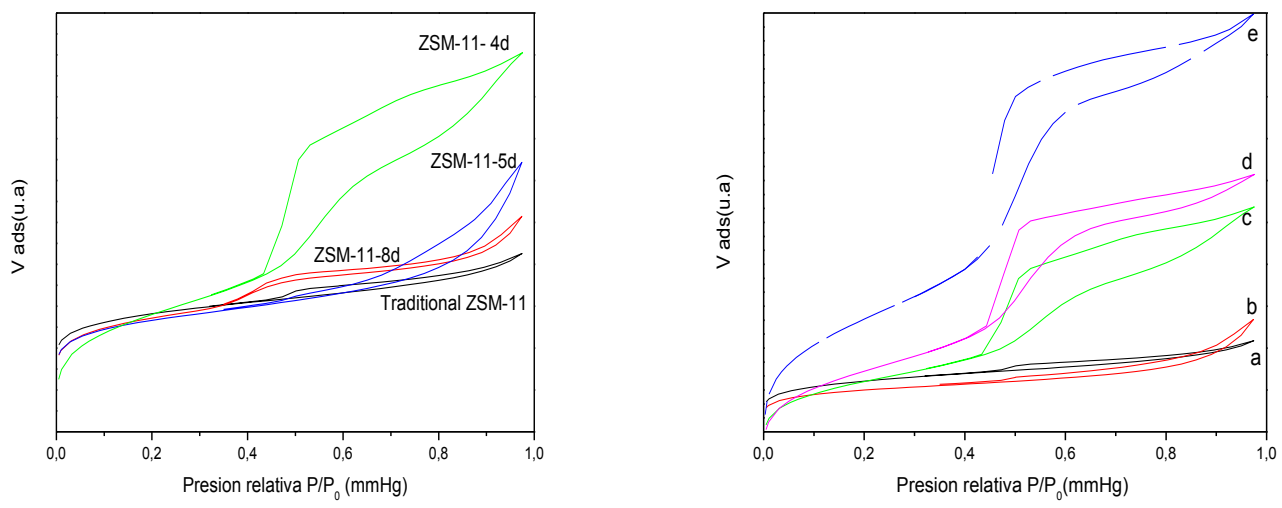

Figura 4. Isotermas de adsorción y desorción de $\mathrm{N}_{2}$. A) Efecto del período de cristalización. B) Efecto del contenido de CTAB: a) ZSM-11 Tradicional, b) ZSM-11-4d-0.4 c) ZSM-11-4d-0.76 d) ZSM-11-4d-1 y e) ZSM-11-4d-1.5.

Los resultados de este análisis determinaron que la zeolita ZSM-11 tradicional presentó isotermas tipo I, mientras que las muestras jerarquizadas exhibieron isoterma combinada tipo I y IV con variación en los loops de histéresis, dependiendo del tiempo de cristalización y del contenido de CTAB. Estos loops de histéresis corresponden el fenómeno de condensación capilar característicos de los materiales mesosporosos, lo que confirma la generación de mesoporosos adicionales en las zeolitas microporosas. En la Tabla 2 se exponen las propiedades texturales de las zeolitas micro/mesoporosas acorde al contenido de CTAB.

Tabla 2. Propiedades texturales de las zeolitas jerarquizadas acorde al contenido de CTAB

\begin{tabular}{lcccccc}
\hline Zeolitas & $\begin{array}{l}\mathrm{S}_{\text {BET }} \\
\left(\mathrm{m}^{2} / \mathrm{g}\right)\end{array}$ & $\begin{array}{l}\mathrm{S}_{\text {EXT }} \\
\left(\mathrm{m}^{2} / \mathrm{g}\right)\end{array}$ & $\begin{array}{c}\mathrm{S}_{\text {Micro }} \\
\left(\mathrm{m}^{2} / \mathrm{g}\right)\end{array}$ & $\begin{array}{l}\mathrm{V}_{\text {Micro }} \\
\left(\mathrm{cm}^{3} / \mathrm{g}\right)\end{array}$ & $\begin{array}{l}\mathrm{V}_{\text {Meso }} \\
\left(\mathrm{cm}^{3} / \mathrm{g}\right)\end{array}$ & $\begin{array}{l}\mathrm{V}_{\text {Total }} \\
\left(\mathrm{cm}^{3} / \mathrm{g}\right)\end{array}$ \\
\hline ZSM-11 T & 282,89 & 85,20 & 197,68 & 0,129 & 0,066 & 0,195 \\
ZSM-11-4d-0.4 & 264,35 & 72,05 & 192,30 & 0,147 & 0,096 & 0,243 \\
ZSM-11-4d-0.76 & 320,11 & 212,88 & 107,23 & 0,073 & 0,310 & 0,383 \\
ZSM-11-4d-1 & 370,13 & 300,82 & 69,30 & 0,047 & 0,376 & 0,423 \\
ZSM-11-4d-1.5 & 539,25 & 457,15 & 82,10 & 0,044 & 0,579 & 0,623 \\
\hline
\end{tabular}

El volumen total y el de mesoporos, así como el área superficial específica (BET) aumentaron cuando se incrementó la cantidad de CTAB de 0,4 a 1,5 g, para un mismo tiempo de cristalización (4 días). Este resultado sugiere que la formación de mesoporosidad en las zeolitas podría ajustarse modificando la cantidad de CTAB incorporado en el gel de síntesis.

Por su parte, en la Tabla 3 se muestran las propiedades texturales de las zeolitas sintetizadas acorde al tiempo de cristalización.

Tabla 3. Propiedades de las zeolitas jerarquizadas obtenidas en distintos períodos de cristalización

\begin{tabular}{lcccrrr}
\hline Zeolitas & $\begin{array}{l}\mathrm{S}_{\text {BET }} \\
\left(\mathrm{m}^{2} / \mathrm{g}\right)\end{array}$ & $\begin{array}{l}\mathrm{S}_{\mathrm{EXT}} \\
\left(\mathrm{m}^{2} / \mathrm{g}\right)\end{array}$ & $\begin{array}{l}\mathrm{S}_{\text {Micro }} \\
\left(\mathrm{m}^{2} / \mathrm{g}\right)\end{array}$ & $\begin{array}{l}\mathrm{V}_{\text {Micro }} \\
\left(\mathrm{cm}^{3} / \mathrm{g}\right)\end{array}$ & $\begin{array}{c}\mathrm{V}_{\text {Meso }} \\
\left(\mathrm{cm}^{3} / \mathrm{g}\right)\end{array}$ & $\begin{array}{l}\mathrm{V}_{\text {Total }} \\
\left(\mathrm{cm}^{3} / \mathrm{g}\right)\end{array}$ \\
\hline ZSM-11 T & 282,89 & 85,20 & 197,68 & 0,129 & 0,066 & 0,195 \\
ZSM-11-8d & 299,65 & 111,67 & 187,98 & 0,114 & 0,133 & 0,247 \\
ZSM-11-5d & 280,7 & 100,37 & 180,33 & 0,117 & 0,175 & 0,292 \\
ZSM-11-4d & 320,11 & 212,88 & 107,23 & 0,073 & 0,31 & 0,383 \\
\hline
\end{tabular}

Como se aprecia en la tabla, el tiempo de cristalización tiene un efecto importante sobre las propiedades texturales, puesto que el volumen de mesoporos, así como el área específica BET se incrementan a medida que disminuye el tiempo de cristalización.

En cuanto a la composición elemental de los materiales preparados, por medio de la técnica analítica de ICP se determinó que la relación molar Si/Al de las zeolitas sintetizadas fue próxima a 17, en todos los casos.

Por su parte, en las imágenes de SEM (no presentadas en este trabajo) se aprecia que la morfología de los cristales se ve modificada con la incorporación del surfactante y con el tiempo de cristalización. Cuando se incorpora CTAB en el gel de 
síntesis, se observan los cristales hexagonales propios de las ZSM-11 tradicional y la aparición de barras hexagonales, indicando la coexistencia de una fase adicional. Sin embargo, en las imágenes de las zeolitas ZSM-11-4d-1 y ZSM-11-4d1.5 se aprecia la aparición de un componente amorfo característico de los materiales mesoporosos. La presencia de esta fase amorfa se ve incrementada con el aumento del contenido de surfactante catiónico en la síntesis.

Finalmente se presentan los resultados de DRX e Isotermas de adsorción y desorción de $\mathrm{N}_{2}$ para los materiales obtenidos a partir de la desilicación de las zeolitas ZSM-11 en función de la sal empleada para este fin (Figura 5 A y B).
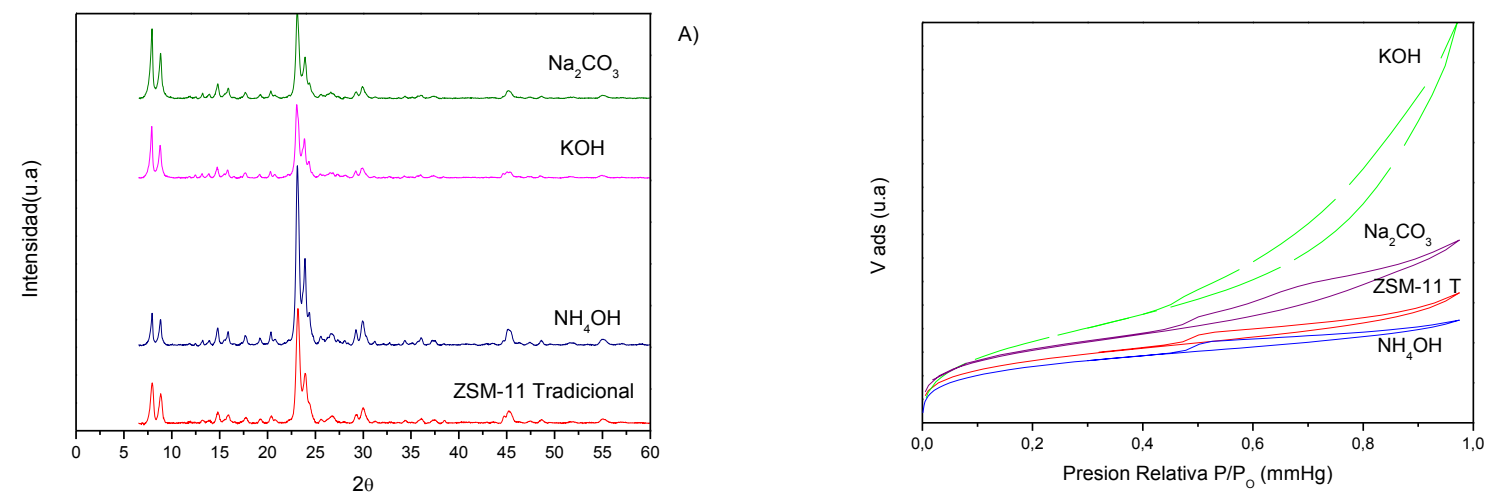

B)

Figura 5. A) Patrones de difracción de las muestras jerarquizadas. B) Isotermas de adsorción y desorción de $\mathrm{N}_{2}$.

Como se observa en la Figura 5 A, las muestras ZSM-11 jerarquizadas poseen la estructura cristalina propia de este tipo de materiales. Lo interesante en este punto es que a partir de los resultados presentados en la Figura 5 B se corrobora la presencia de isotermas combinadas del tipo I y IV con variación en el loop de histéresis, en función de la base empleada, a excepción del material tratado con la solución alcalina $0,3 \mathrm{M}$ de $\mathrm{NH}_{4} \mathrm{OH}$, el cual exhibe una isoterma tipo I característica de los materiales con estructura microporosa.

De este modo, los resultados obtenidos de las distintas caracterizaciones realizadas a los materiales confirmaron la creación de porosidad adicional en las zeolitas ZSM-11 microporosas.

Los materiales jerarquizados preparados por síntesis directa y tratamientos post síntesis serán modificados mediante la incorporación de distintas especies activas para evaluar su perfomace catalítica en la reacción de oxidación de glicerol para obtener ácido láctico y dihidroxiacetona.

\section{Referencias}

[1] Liu, H., Zhang, S., Xie, S., Zhang, W., Xin, W., Liu, S., Xu, L. (2018). Synthesis, characterization, and catalytic performance of hierarchical ZSM-11 zeolite synthesized via dual-template route. Chinese Journal of Catalysis 39, 167180.doi.org/10.1016/S1872-2067(17)62984-X.

[2] Chen, H. L., Ding, J., \& Wang, Y. M. (2014). One-step synthesis of mesoporous ZSM-11 composites through a dualtemplate method. New Journal of Chemistry, 38(1), 308-316. doi.org/10.1039/C3NJ00785E.

[3] Xue, T., Liu, H., Zhang, Y., Wu, H., Wu, P., He, M. (2017). Synthesis of ZSM-5 with hierarchical porosity: In-situ conversion of the mesoporous silica-alumina species to hierarchical zeolite. Microporous and Mesoporous Materials, 242, 190-199. doi.org/10.1016/j.micromeso.2017.01.021.

[4] Y.Ma, J. Hu, L. Jia, Z. Li, Q. Kan, S.Wu. (2013). Synthesis, characterization and catalytic activity of a novel mesoporous ZSM-5 zeolite.Materials Research Bulletin 48, 1881-1884. doi.org/10.1016/j.materresbull.2013.01.014.

[5] H. Tao, C. Li, J. Ren, Y. Wang, G. Lu (2011). Synthesis of mesoporous zeolite single crystals with cheap porogens. Journal of Solid State Chemistry 184, 1820- 1827. doi.org/10.1016/j.jssc.2011.05.023

[6] Liu, H., Liu,S., Xie, S., Song, C., Xin, W., Xu, L. (2015). Effect of Desilication on the Performance of Hierarchical ZSM-11 Catalysts for Alkylation of Benzene with Dimethyl Ether. Catalysis Letters, 145(11), 1972-1983. doi.org/10.1007/s10562-015-1589-1.

[7] Groen, J. C., Moulijn, J. A., \& Pérez-Ramírez, J. (2007). Alkaline posttreatment of MFI zeolites. From accelerated screening to scale-up. Industrial \& engineering chemistry research, 46(12), 4193-4201. doi.org/10.1021/ie061146v. 